\title{
Évaluation d'un système de mesure des pertes de contaminants agricoles par ruissellement et drainage souterrain en régions froides
}

\section{Evaluation of a measurement system for agricultural contaminant losses by runoff and subsurface drainage in cold areas}

\author{
M. Goulet, J. Gallichand, M. Duchemin et M. Giroux
}

Volume 18, numéro 4, 2005

URI : https://id.erudit.org/iderudit/705570ar

DOI : https://doi.org/10.7202/705570ar

Aller au sommaire du numéro

Éditeur(s)

Université du Québec - INRS-Eau, Terre et Environnement (INRS-ETE)

ISSN

0992-7158 (imprimé)

1718-8598 (numérique)

Découvrir la revue

Citer cet article

Goulet, M., Gallichand, J., Duchemin, M. \& Giroux, M. (2005). Évaluation d'un système de mesure des pertes de contaminants agricoles par ruissellement et drainage souterrain en régions froides. Revue des sciences de l'eau / Journal of Water Science, 18(4), 507-519. https://doi.org/10.7202/705570ar

\section{Résumé de l'article}

Cette note technique présente un système automatique de mesure des pertes de polluants agricoles par ruissellement et drainage souterrain pouvant opérer durant la période estivale et la fonte nivale. Le système de mesure du ruissellement est composé d'une trappe à sédiments, d'un déversoir en $\mathrm{V}$ et d'un système automatique de mesure du débit et de contrôle de l'échantillonnage. Le système de mesure du drainage souterrain consiste en un auget à bascule, relié à un compteur d'impulsions, et un partiteur de débit. Ces systèmes ont été utilisés sur neuf parcelles expérimentales pour mesurer les masses de contaminants agricoles perdues par ruissellement et drainage souterrain. La hauteur totale de ruissellement durant les deux années $(4,6 \mathrm{~mm}$ $\mathrm{an}^{-1}$ ) a été faible comparée au drainage $\left(227 \mathrm{~mm} \mathrm{an}^{-1}\right)$. Soixante-seize pourcent du ruissellement annuel s'est produit durant la fonte des neiges. Les principaux avantages de ce système sont de pouvoir mesurer simultanément les volumes et les débits de ruissellement tout en prélevant des échantillons d'eau. 


\title{
Évaluation d'un système de mesure des pertes de contaminants agricoles par ruissellement et drainage souterrain en régions froides
}

\author{
Evaluation of a measurement system for \\ agricultural contaminant losses by runoff and \\ subsurface drainage in cold areas
}

\section{GOULET ${ }^{1}$, J. GALLICHAND ${ }^{1 *}$, M. DUCHEMIN² ${ }^{2}$ M. GIROUX ${ }^{2}$}

Reçu le 11 mars 2004, accepté le 11 janvier 2005* .

\section{SUMMARY}

This technical note presents an automatic measurement system for measuring losses of agricultural pollutants in surface runoff and subsurface drainage outflow. This system can be operated during rainfall events and snowmelt periods. The runoff system was composed of a sediment trap, a $V$-noteh weir and an automatic system for flow measurement and sampling. The subsurface drainage system consisted of a tipping bucket, connected to a pulse counter, and a sampling unit. This system was used on nine experimental plots for the measurement of pollutant losses by surface runoff and subsurface drainage. The total depth of surface runoff measured was low for the two years of measurement $\left(4.6 \mathrm{~mm}^{-1}\right)$ compared to subsurface drainage $\left(227 \mathrm{~mm} \mathrm{y}^{-1}\right)$. Seventy six percent of the annual surface runoff occurred during the snowmelt period. The principal advantage of this system is to measure runoff volume and flow while taking water samples.

Keywords: measurement system, runoff, subsurface drainage, pollutants losses, snowmelt.

\section{RÉSUMÉ}

Cette note technique présente un système automatique de mesure des pertes de polluants agricoles par ruissellement et drainage souterrain pouvant opérer durant la période estivale et la fonte nivale. Le système de mesure du ruissellement est composé d'une trappe à sédiments, d'un déversoir en $V$ et d'un système automatique de mesure du débit et de contrôle de l'échantillonnage. Le système de mesure du drainage souterrain consiste en un auget à bascule, relié à un compteur d'impulsions, et un partiteur de débit. Ces sys-

1. Département des Sols et de Génie Agroalimentaire, Université Laval, Québec, PQ, G1K 7P4.

2. Institut de Recherche et de Développement en Agro-environnement Inc. (IRDA) Complexe scientifique, 2700, rue Einstein, Local D.1.110, Sainte-Foy, G1P 3 W8.

* Correspondance: jacques.gallichand@sga.ulaval.ca

** Les commentaires seront reçus jusqu'au 30 août 2006. 
tèmes ont été utilisés sur neuf parcelles expérimentales pour mesurer les masses de contaminants agricoles perdues par ruissellement et drainage sonterrain. La hauteur totale de ruissellement durant les deux années $\left(4,6 \mathrm{~mm} \mathrm{an}^{-1}\right)$ a été faible comparée au drainage $\left(227 \mathrm{~mm} \mathrm{an}^{-1}\right)$. Soixanteseize pourcent du ruissellement annuel s'est produit durant la fonte des neiges. Les principaux avantages de ce système sont de pouvoir mesurer simultanément les volumes et les débits de ruissellement tout en prélevant des échantillons d'eau.

Mots clés : système de mesure, drainage souterrain, ruissellement, fonte nivale, polliuant agricole.

\section{1 - INTRODUCTION}

Plusieurs systèmes de mesure des pertes de contaminants agricoles en régions froidies sont décrits dansila littérature (CHOW et al., 2000 ; GANGBAZO et al., 1997 ; HANSEN et al., 2000; ; JAMIESON et al., 2003). Ces systèmes nécessitent de quantifier le débit et la concentration des contaminants pouvant être transportés sous forme dissoute ou particulaire par drainage souterrain et par ruissellement.

L'auget à bascule avecenregistreur de données convient pour les débits faibles et continus comme ceux observés en drainage souterrain mais devient moins précis pour les gramds débits correspondant à une fréquence de bascules élevée. Pour le ruissellement, un système à section d'écoulement critique avec senseur de nivatu plus adapté (KUYKENDALL et al., 1999 ; JAMIESON et al., 2003). Le prélèvement d'échantillons d'eau peut se faire à l'aide de partiteurs de débits et dontillonneurs programmables (LAPP et al., 1998). Les partiteurs de débits; prouvant toutefois être obstrués par les sédiments (CHOW et al., 2000 ; GANGBAZO et a/., 1997), sont mieux adaptés à la mesure de l'eau de drainage souterrain, alors que l'échantillonneur programmable présente l'avantage de pouvoir fonctionner en présence de sédiments (STONE et al., 2000).

En régions froides avec accumulation de neige, le ruissellement est causé par deux mécanismes distincts : la fonte nivale printanière et les précipitations liquides, dites estivales, survenant entre les mois d'avril et novembre (LAPP et al., 1998). L'écoulement souterrain s'étend sur toute l'année alors que la fonte nivale survient durant seulement deux à trois semaines, mais la perte de contaminants qui y est associée est importante (JAMIESON et al. 2003 ; GANGBAZO et al., 1997).

L'objectif de cette note technique est de présenter et d'évaluer un système automatique de mesure des pertes annuelles de contaminants agricoles par ruissellement et drainage souterrain, pouvant opérer en périodes estivale et de fonte nivale. Un exemple de résultats obtenus est présenté pour le phosphore (P) et les sédiments. 


\section{2 - MATÉRIELS ET MÉTHODES}

\subsection{Site expérimental}

Le dispositif expérimental était constitué de neuf parcelles de 0,11 à 0,22 ha chacune, avec des pentes allant de 0,5 à $2,8 \%$ et lacalisées dans les champs 35 et 58 de la ferme expérimentale de l'Institut de Recherche et de Développement en Agroenvironnement à Saint-Lambert-de-Lauzon, $10 \mathrm{~km}$ au sud de Québec. Le sol de ces deux champs est un loam limoneux de la série Le Bras (ROMPRÉ, 1985); la teneur en P-Mehlich-3 variait de 13 à $58 \mathrm{mg} \mathrm{kg}^{-1}$ et le taux de saturation en $P$ de 1,3 à $4,3 \%$ (GIROUX et TRAN, 1996). Chaque parcelle était équipée de deux systèmes de mesure et d'échantillonnage : un pour le nuissellement et l'autre pour le drainage souterrain. La figure 1 illustre les parcelles expérimentales du champ 35 ; le champ 58 était disposé de la même manière mais comptait cinq parcelles. Des mesures de débit et de concentration ont été effectuées en 2001 et 2002 . Les quatre parcelles du champ 35 et une parcelle du champ 58 étaient en orge (Hordeum vulgare L.) sous-ensemencé en prairie en 2001 et en prairie en 2002 . Les quatre autres parcelles du champ 58 étaient en canola (Brassica napus L.) en 2001 et en maïs-grain (Zea mays L.) en 2002. Le climat de cette région est tempéré froid avec des normales climatiques annuelles de $1126 \mathrm{~mm}$ pour la précipitation totale, dont $252 \mathrm{~mm}$ sous forme de précipitation solide, et une température moyenne de l'air de $4,3^{\circ} \mathrm{C}$ (ENVIRONNEMENT CANADA, 2004). Les précipitations liquides ont été mesurées du début avril à la fin novembre de chaque année par deux pluviomètres à bascule installés sur le site. Ces données ont été complétées par celles de la station de Scott (ENVIRONNEMENT CANADA, 2004), localisẻe à $7 \mathrm{~km}$ au sud du site expérimental, de façon à obtenir des années complètes (janvier à décembre) de précipitations liquides et solides. L'évapotranspiration de référence a été calculée du $1^{\text {er }}$ avril au 30 novembre avec l'équation FAO56 (FAO Penman-Monteith) à l'aide du logiciel REF-ET (ALLEN, 2000), et multipliée par les coefficients de cultures appropriés pour obtenir l'évapotranspiration réelle.

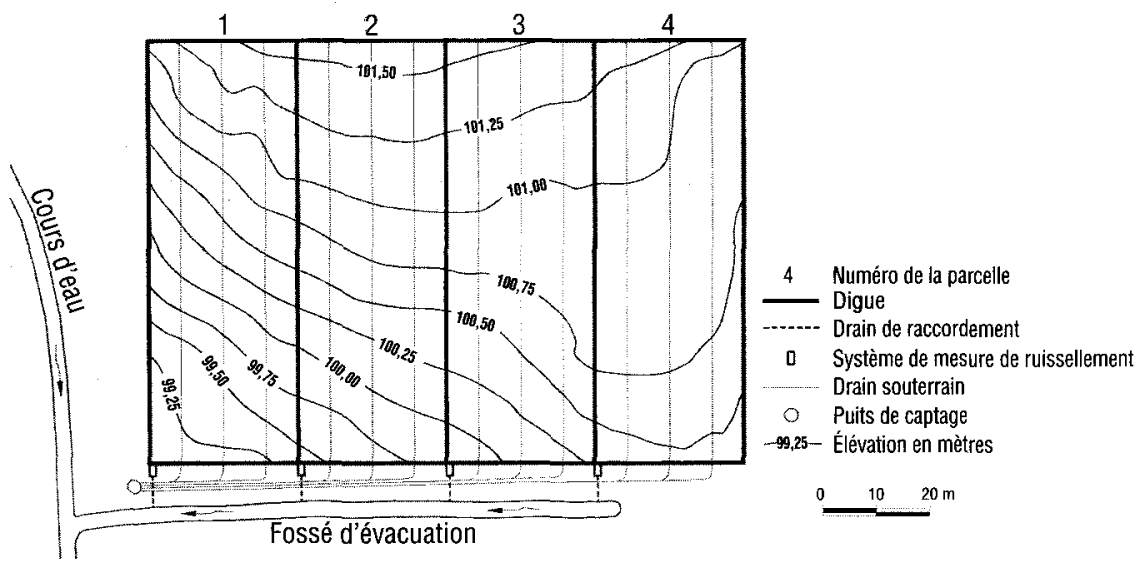

Figure 1 Champ expérimental $\mathrm{n}^{\circ} 35$ et localisation des systèmes de mesure.

Experimental field plot $n^{\circ} 35$ and location of measurement systems. 


\subsection{Système de mesure du ruissellement}

Le système de mesure et d'échantillonnage du ruissellement est montré à la figure 2. L'eau d'une parcelle converge vers le cabanon (A), entre par l'ouverture d'admission de l'eau $(B)$, est retenue par un contenant de rétention (C) dans lequel se trouve la crépine d'aspiration (D) de l'échantillonneur (modèle 900, AMERICAN SIGMA INC., Loveland, CO). Du contenant de rétention, l'eau se déverse dans la trappe à sédiments $(E)$ où le niveau d'eau est déterminé en continu par une sonde ultrasonique ( $F$ - modèle PZ-12, GREYLINE INSTRUMENTS INC., Massena, NY) qui transmet à intervalle de 2 s un signal analogique au niveaumètre-débitmètre ( $\mathrm{G}$ - modèle SLT32, Greyline Instruments Inc.), lequel effectue trois opérations:

1) affichage du volume total d'eau ruisselée ;

2) transmission à intervalle de $20 \mathrm{~s}$ d'un signal analogique à l'enregistreur de débit ( $\mathrm{H}$ - modèle OWL-500, ACR System Inc., Surrey, BC) qui le convertit en valeur numérique de débit, et

3) envoi d'un signal déclenchant la prise d'un échantillon de $100 \mathrm{ml}$ lorsque le volume de consigne de $5 \mathrm{I}$ est atteint. En aval du déversoir en $\mathrm{V}$ de $30 \mathrm{~cm}$ de hauteur (I), l'eau est évacuée par un drain $(\mathrm{J})$ et un fossé $(\mathrm{K})$. Tous les appareils électroniques étaient alimentés par des batteries marines $12 \mathrm{~V}(\mathrm{~L})$. Chaque parcelle était entourée de digues triangulaires en terre, semées de gazon et tondues régulièrement, pour éviter l'écoulement d'une parcelle à une autre et pour diriger l'eau vers le système de mesure.

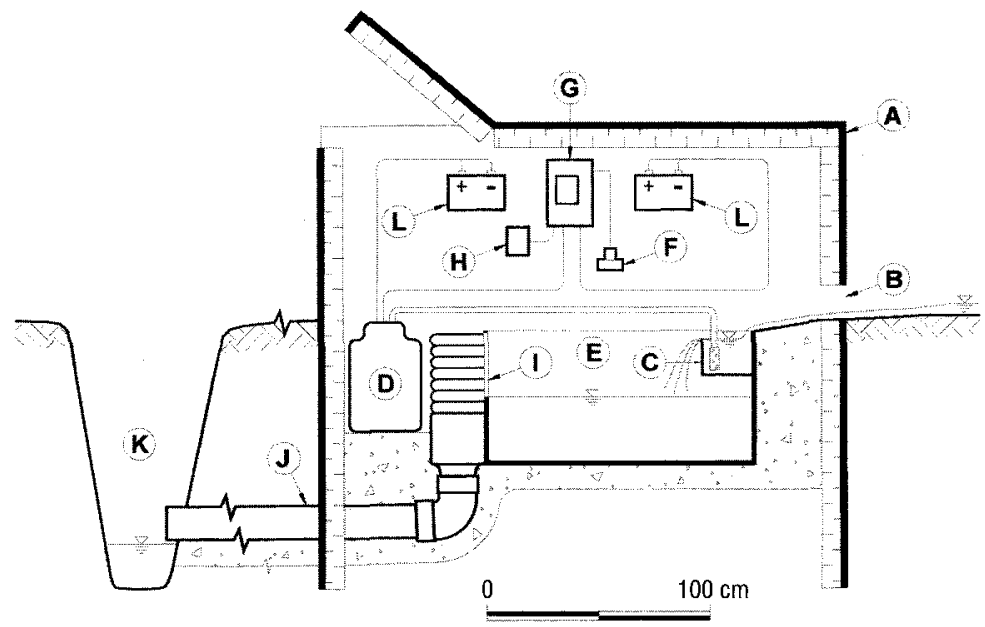

Figure 2 Système de captage, de mesure et d'échantillonnage du ruissellement. A - cabanon isolé ; B - ouverture d'admission ; C - contenant de rétention ; $\mathrm{D}$ - échantillonneur ; $\mathrm{E}$ - trappe à sédiments ; $\mathrm{F}$ - sonde ultrasonique ; $\mathrm{G}$ - niveaumètre-débitmètre ; $\mathrm{H}$ - enregistreur ; I - déversoir ; $\mathrm{J}$ - drain d'évacuation ; $\mathrm{K}$ - fossé ; $\mathrm{L}$ - batteries marine.

Runoff system for flow measurement and sampling.

$A$ - insulated shed; $B$ - opening for runoff; $C$ - retention container; $D$ - sampler; $E$ - sediment trap; $F$ - ultrasonic water level sensor; $G$ - control unit; $H$ - datalogger; $I$ - V-notch weir; $J$ - outlet to surface drain; $K$ - ditch; $L$ - marine battery. 
Pour chaque événement-parcelle (i.e. un événement de précipitation ayant généré du ruissellement sur une parcelle), le volume total d'eau enregistré par le totalisateur était noté et un échantillon d'eau de 1 I prélevé dans le réservoir de l'échantillonneur suite à une agitation préalable servant à remettre les sédiments en suspension. L'enregistreur de données a fourni les débits et la durée de ruissellement permettant de vérifier l'exactitude du volume total enregistré par le totalisateur. Tous les échantillons d'eau ont été conservés à $4{ }^{\circ} \mathrm{C}$ et analysés selon les méthodes d'analyse reconnues au Québec pour le $P$ total (APHA, 1985a ; TECHNICON, 1973) et les matières en suspension (MES) (APHA, 1985b). Pour chaque événement-parcelle, la masse de $P$ total perdue a été obtenue en multipliant le volume d'eau ruisselé de la parcelle par la concentration en $\mathrm{P}$ total de l'échantillon proportionnel de l'échantillonneur.

À la fin de chaque saison, les trappes à sédiments ont été vidées, les sédiments séchés à $105^{\circ} \mathrm{C}$, pesés et analysés pour le $P$ total. Des analyses granulométriques ont été effectuées, par la méthode de l'hydromètre (SHELDRICK et WANG, 1993), sur les sédiments déposés dans la trappe à sédiments de chaque parcelle au cours de la saison ainsi que sur le sol de surface des deux champs, soit deux échantillons composites par parcelle prélevés en mai 2001 dans la couche 0-15 cm. Des analyses granulométriques ont aussi été effectuées sur le sol contenu dans les échantillonneurs du champ 58 en septembre 2001.

\subsection{Système de mesure et d'échantillonnage du drainage souterrain}

Chaque parcelle était drainée par quatre latéraux de $10 \mathrm{~cm}$ de diamètre (espacement $7 \mathrm{~m}$, profondeur variant de 0,92 à $1,5 \mathrm{~m}$ et pente moyenne de $1 \%)$ (figure 1). Un drain tampon à la limite entre deux parcelles se déchargeait directement dans un ruisseau, assurant l'indépendance hydrologique des parcelles (non illustré). L'écoulement des trois drains souterrains de chaque parcelle était capté par un collecteur (A - figure 3 ) et dirigé vers un puits de captage circulaire en acier galvanisé $(B)$ où le volume de drainage était mesuré par un auget à bascule $(C)$ de $1 \mathrm{~L}$ relié à un compteur d'impulsions ( $D$ - modèle 79998D-110, Veeder-Root, Simsbury, CT) lu à chaque semaine. Le dispositif d'échantillonnage était formé d'un tube de plastique de $5 \mathrm{~mm}$ dirigeant une fraction de l'eau du collecteur dans un récipient de $4 L(E)$ à partir duquel un échantillon de $500 \mathrm{ml}$ était prélevé à chaque semaine. Les échantillons d'eau étaient conservés à $4{ }^{\circ} \mathrm{C}$ et analysés pour le $\mathrm{P}$ total. Les mesures du drainage souterrain effectuées en continu durant les deux années ont été regroupées sur une base hebdomadaire. 


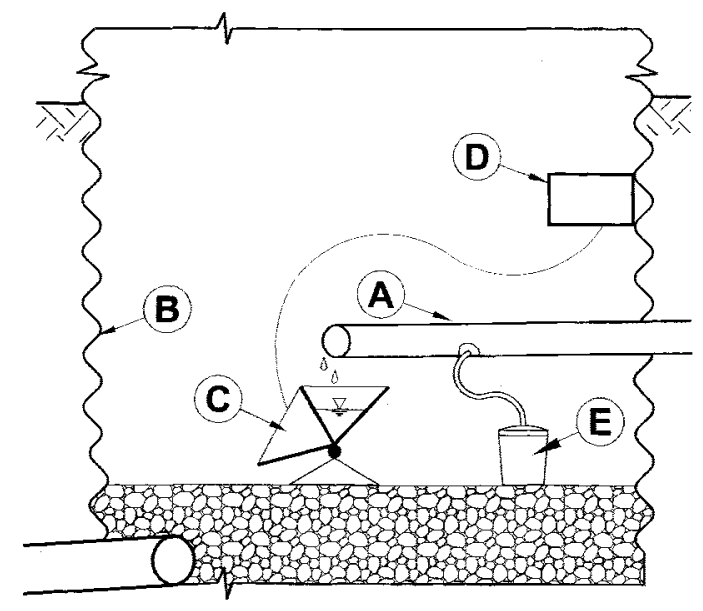

Figure 3 Système de captage, de mesure et d'échantillonnage du drainage souterrain.

A - collecteur ; B - puits de captage circulaire ; C - auget à bascule ; D - compteur d'impulsions ; $\mathrm{E}$ - récipient d'échantillonnage.

Subsurface drainage system for flow measurement and sampling.

$A$ - collector pipe; $B$ - manhole; $C$ - tipping bucket; $D$ - pulse counter; E - sampling container.

\section{3 - RÉSULTATS ET DISCUSSION}

\subsection{Caractérisation des précipitations}

La hauteur totale des précipitations liquides mesurée sur le site entre avril et novembre a été de $644 \mathrm{~mm}$ en 2001 et $652 \mathrm{~mm}$ en 2002, valeurs près de la normale climatique locale de $682 \mathrm{~mm}$ pour la même période (station de Scott ENVIRONNEMENT CANADA, 2004). En combinant les données locales et celles de Scott, on obtient une précipitation totale de $976 \mathrm{~mm}$ en 2001 et de $978 \mathrm{~mm}$ en 2002. La précipitation solide a résulté en une hauteur de neige au sol de 64 et $35 \mathrm{~cm}$ au 31 mars 2001 et 2002, respectivement. Les événements pluvieux observés correspondaient à des périodes de récurrence inférieure à 2 ans, à l'exception de deux événements (3,25 et 2,43 ans). Ainsi, le système de mesure et d'échantillonnage du ruissellement n'a pu être testé pour des intensités très élevées de précipitation. Des 155 événements de précipitation survenus en 2001 et 2002, 18 seulement ont causé du ruissellement dans au moins une des neuf parcelles : 11 événements en 2001 (61 événements-parcelles), et sept en 2002 (44 événements-parcelles).

\subsection{Système de mesure de ruissellement}

Pour les deux années, les systèmes de mesure et d'échantillonnage étaient opérationnels avant le début de la fonte des neiges, i.e. le 5 avril en 2001 et le 31 mars en 2002. Au cours des deux hivers, quelques événements de précipi- 
tation liquide (hauteur maximale de $19 \mathrm{~mm}$ ) sont survenus sur un couvert neigeux supérieur à $10 \mathrm{~cm}$. Des visites au site durant cette période n'ont montré aucune trace de ruissellement. Après la mi-novembre, les systèmes de mesure de ruissellement ont été débranchés en raison du sol gelé, du couvert de neige et de l'absence de ruissellement.

Les observations effectuées durant la fonte nivale de 2001 ont mis en évidence l'importance d'une mesure précise des faibles débits. En effet, durant cette période, le débit de ruissellement était d'environ $0,05 \mathrm{~L} \mathrm{~s}^{-1}$ en moyenne. Pour un déversoir triangulaire de $30^{\circ}$, cette valeur correspond à une hauteur de $2,7 \mathrm{~cm}$ et à une précision de $\pm 0,02 \mathrm{~L} \mathrm{~s}^{-1}( \pm 2 \mathrm{~mm})$. Pour augmenter la précision de mesure du débit, les déversoirs de $30^{\circ}$ ont été changés au début mai 2001 pour des déversoirs de $10^{\circ}$, diminuant l'erreur à $\pm 0,01 \mathrm{~L} \mathrm{~s}^{-1}$. Ce changement s'est avéré nécessaire considérant la grande proportion du ruissellement survenant à des débits inférieurs à $1 \mathrm{~L} \mathrm{~s}^{-1}$. La capacité du déversoir de $10^{\circ}\left(6,2 \mathrm{~L} \mathrm{~s}^{-1}\right)$ n'a jamais été dépassée durant les deux années de mesure.

Les trappes à sédiments ont été construites en contreplaqué, avec joints scellés au silicone et enduites d'une résine marine. Des fuites ont été observées dans une des trappes durant la fonte des neiges 2002, résultant en la perte des données de cette période et le rejet de cette année-parcelle. Pour corriger ce problème, l'intérieur de toutes les trappes a été recouvert d'un polyéthylène $0,41 \mathrm{~mm}$, prévenant toutes fuites éventuelles. Des installations futures devraient prévoir des trappes en fibre de verre, plastique ou acier.

Ces systèmes de mesure avaient été conçus pour une opération autonome. Toutefois, une présence fréquente au champ s'est avérée requise pour s'assurer de la fiabilité des mesures, surtout durant la période de fonte des neiges où il peut y avoir formation de glace dans la trappe à sédiments. Dans notre cas, tous les appareils fonctionnaient avec des batteries marines changées chaque semaine. L'utilisation d'appareils fonctionnant sur le réseau d'alimentation électrique permettrait de réduire le nombre d'heures requis pour le fonctionnement du système et aussi d'installer du chauffage pour prévenir la formation de glace au niveau du déversoir.

\subsection{Système de mesure de l'eau de drainage}

Étant donné la régularité du débit, l'absence de gel et la faible concentration en sédiments, le système de mesure de l'eau de drainage est plus simple que celui pour le ruissellement, et son opération a présenté moins de difficultés. Aux printemps 2001 et 2002, l'élévation rapide du niveau de l'eau dans le ruisseau adjacent a causé une remontée de l'eau dans le puits de captage du champ 35 résultant en la perte de deux jours de données à chaque année. Ces inondations sont survenues au début de la fonte des neiges alors que le sol était encore gelé et que le débit des drains était de l'ordre de $4,5 \mathrm{~mm} \mathrm{j}^{-1}$. Les données manquantes ont été estimées par régression à partir des données du champ $58(R=0,98$ en 2001 et 0,80 en 2002). Cet inconvénient aurait pu être évité par l'installation d'une pompe électrique si le branchement au secteur avait été possible.

Le système d'échantillonnage des eaux de drainage est constitué d'un orifice prélevant un volume d'eau proportionnel à l'épaisseur de l'écoulement dans le drain. La fréquence d'échantillonnage hebdomadaire a été sélectionnée 
suite aux résultats d'une étude préliminaire qui n'a montré aucune différence entre la moyenne hebdomadaire de la concentration en $\mathrm{P}$ basée sur un ou deux échantillons par semaine.

\subsection{Ruissellement, drainage souterrain et perte de phosphore}

Seules les années-parcelles pour lesquelles les données étaient complètes (fonte des neiges, ruissellement et drainage souterrain) ont été retenues pour l'analyse, soit 3 années-parcelles en 2001 et 8 en 2002. Les années-parcelles incomplètes sont le résultat de délais de construction et de pannes du système de mesure. Pour les deux années à l'étude, la fonte nivale s'est produite durant deux à trois semaines en avril avec une durée de ruissellement allant de $2 \mathrm{~h}$ à $24 \mathrm{~h}$. Le ruissellement estival s'est produit de la fin avril à la mi-novembre pour des durées de $8 \mathrm{~min}$ à $3 \mathrm{~h} 45$. Le ruissellement de la fonte nivale représente en moyenne $89 \%$ du ruissellement annuel, soit une hauteur de $4,1 \mathrm{~mm}$ (tableau 1). En 2001, $64 \%$ de tous les événements de ruissellement se sont produits des débits inférieurs à $1,0 \mathrm{~L} \mathrm{~s}^{-1}$ comparés à $98 \%$ en 2002. Le débit maximal observé a été $4,6 \mathrm{~L} \mathrm{~s}^{-1}$ en 2001 et $1,8 \mathrm{~L} \mathrm{~s}^{-1}$ en 2002 . Le débit de pointe de $4,6 \mathrm{~L} \mathrm{~s}^{-1}$ n'a duré que $80 \mathrm{~s}$ et correspond à l'événement de précipitation d'une récurrence de 3,25 ans. Le débit de ruissellement n'a jamais dépassé la capacité du déversoir (angle de $10^{\circ}$ et $30 \mathrm{~cm}$ de hauteur), soit $6,2 \mathrm{~L} \mathrm{~s}^{-1}$ correspondant à une récurrence de deux ans telle que calculée selon la méthode du numéro de courbe modifiée pour le Québec (MONFET, 1979). Ces observations ont mis en évidence la nécessité d'un système de mesure capable de déterminer à la fois les faibles débits de fonte des neiges et les débits plus élevés des orages d'été.

Compte tenu du retard de l'écoulement souterrain par rapport au ruissellement, on ne peut distinguer l'écoulement souterrain des périodes fonte nivale et estivale. Le drainage souterrain s'est produit en continu durant les deux années avec des pointes printanières (avril à mai) et automnales (septembre à novembre). L'épaisseur de drainage souterrain a été en moyenne de $227 \mathrm{~mm}$ (tableau 1), soit $98 \%$ de l'écoulement total (ruissellement et souterrain). Le débit maximal a été de $17 \mathrm{~mm} \mathrm{j}^{-1}$ en avril 2001. La répartition des pertes de $\mathrm{P}$ total suit celle de l'eau, i.e. $517 \mathrm{~g} \mathrm{ha}^{-1}$ (93\%) par drainage souterrain et $26,3 \mathrm{~g} \mathrm{ha}^{-1}$ (7\%) par ruissellement (tableau 2).

Tableau 1 Hauteurs annuelles moyennes de ruissellement et de drainage souterrain.

Table 1 Average annual runoff and subsurface drainage depth.

\begin{tabular}{lccccc}
\hline & $\mathbf{n}^{\mathbf{2}}$ & \multicolumn{3}{c}{ Ruissellement } & Drainage \\
\hline & & Fonte nivale & $\begin{array}{c}\text { Estival } \\
(\mathbf{m m})\end{array}$ & Total & \\
\hline 2001 & 3 & 6,4 & 0,8 & 7,2 & 214 \\
2002 & 8 & 3,3 & 0,4 & 3,7 & 231 \\
Moyenne & & 4,1 & 0,5 & 4,6 & 227 \\
\hline
\end{tabular}

z: nombre d'années-parcelles. 
Tableau 2 Pertes annuelles moyennes de phosphore total et de sédiments par ruissellement et drainage souterrain.

Table 2 Average annual losses of total phosphorus and sediment by runoff and subsurface drainage.

\begin{tabular}{|c|c|c|c|c|c|c|}
\hline & \multirow{3}{*}{$n$} & \multicolumn{4}{|c|}{ Ruissellement } & \multirow[t]{3}{*}{ Drainage } \\
\hline & & \multirow[t]{2}{*}{ Trappe $^{\mathrm{Z}}$} & \multicolumn{3}{|c|}{ Échantillonneury } & \\
\hline & & & Fonte nivale & Estival & Total & \\
\hline$P$ total & & & & $\left(g h a^{-1} a n^{-1}\right)$ & & \\
\hline $\begin{array}{l}2001 \\
\quad \text { Champ } 35\end{array}$ & 3 & 0,3 & 10,1 & 13,2 & 23,3 & 365 \\
\hline $\begin{array}{l}2002 \\
\text { Champ } 35 \\
\text { Champ } 58 \\
\text { Moyenne }(n=11)\end{array}$ & $\begin{array}{c}4 \\
4 \\
9,8\end{array}$ & $\begin{array}{c}0,0 \\
26,8 \\
7,1\end{array}$ & $\begin{array}{c}10,1 \\
2,0 \\
19,1\end{array}$ & $\begin{array}{l}12,5 \\
30,2 \\
26,3\end{array}$ & $\begin{array}{l}22,6 \\
32,2 \\
517\end{array}$ & $\begin{array}{l}389 \\
757\end{array}$ \\
\hline Sédiments & & & & & & \\
\hline $\begin{array}{l}2001 \\
\quad \text { Champ } 35\end{array}$ & 3 & 0,5 & 6,6 & $\begin{array}{c}\left(\mathrm{kg} \mathrm{ha}^{-1} \mathrm{an}^{-1}\right) \\
3,9\end{array}$ & 10,5 & $-x$ \\
\hline $\begin{array}{l}2002 \\
\text { Champ } 35 \\
\text { Champ } 58 \\
\text { Moyenne }(n=11)\end{array}$ & $\begin{array}{l}4 \\
4\end{array}$ & $\begin{array}{c}0,0 \\
36,7 \\
12,3\end{array}$ & $\begin{array}{l}1,9 \\
1,4 \\
3,0\end{array}$ & $\begin{array}{c}0,9 \\
32,2 \\
13,1\end{array}$ & $\begin{array}{c}2,7 \\
33,6 \\
16,1\end{array}$ & $\begin{array}{l}- \\
- \\
-\end{array}$ \\
\hline
\end{tabular}

$\mathrm{z}: \mathrm{P}$ total obtenus par la trappe à sédiments à la fin de la saison.

$y: P$ total obtenus par l'échantillonneur pour le ruissellement de fonte des neiges et le ruissellement estival.

$x$ : non applicable.

\subsection{Bilan hydrique}

Un bilan hydrique annuel a été calculé pour valider les mesures de ruissellement et de drainage. La précipitation annuelle totale (solide et liquide) est estimée à $976 \mathrm{~mm}$ en 2001 et à $978 \mathrm{~mm}$ en 2002. La précipitation annuelle totale reçue sur une parcelle devrait être approximativement égale aux pertes par :

1) évapotranspiration réelle,

2) sublimation du couvert neigeux,

3) ruissellement,

4) drainage souterrain, et

5) percolation sous les drains souterrains. L'évapotranspiration réelle du $1^{\mathrm{er}}$ avril au 30 novembre a été de $590 \mathrm{~mm}$ en 2001 et de $559 \mathrm{~mm}$ en 2002 . La sublimation a été estimée à partir du nombre de jours avec couvert neigeux et du taux journalier de sublimation rapporté dans la littérature pour le Québec et l'Ontario, soit de 0,4 à $1,0 \mathrm{~mm} \mathrm{j}^{-1}$ pour la région de Québec (PLAMONDON et al., 1984), 1,0 $\mathrm{mm} \mathrm{j}^{-1}$ pour le sud de l'Ontario (FASSNACHT et al., 1999), et 0,029 à $1,642 \mathrm{~mm} \mathrm{j}^{-1}$ pour l'est de l'Ontario (WILLIAMS, 1959). Nous avons assumé une valeur de sublimation $1,0 \mathrm{~mm} \mathrm{j}^{-1}$ tel que proposé par JAMIESON et al. (2003) pour le Québec. Cette hypothèse résulte en une sublimation annuelle de $158 \mathrm{~mm}$ en 2001 et $141 \mathrm{~mm}$ en 2002. En soustrayant de la précipitation totale les valeurs mesurées (ruissellement, drainage) et estimées (évapotranspiration, sublimation), on obtient entre 7 et $52 \mathrm{~mm}$ pour la percolation sous les drains. Cette valeur apparaît réaliste puisque la pente du terrain et la proximité du cours d'eau peuvent créer un gradient hydraulique local favorable à un écoulement latéral vers le cours d'eau. 


\subsection{Bilan de masse du phosphore}

Un système de mesure des pertes de contaminants agricoles par ruissellement doit pouvoir échantillonner l'ensemble des sédiments transportés par l'eau. Bien qu'il soit possible de déterminer avec précision la granulométrie du sol de surface des parcelles, l'érosion de ce sol se fait de façon sélective et la granulométrie des sédiments atteignant l'exutoire du champ est plus fine que celle du sol de surface (MABIT et al., 2002). Cet enrichissement en particules fines est montré à la figure 4 où l'on constate que les sédiments prélevés par l'échantillonneur contiennent $25 \%$ plus d'argile, $10 \%$ moins de limon et $15 \%$ moins de sable que le sol de surface.

Un problème potentiel est que l'échantillonneur ne puisse prélever l'ensemble des particules atteignant l'exutoire du champ. En utilisant la loi de Stokes (GUPTA, 1997) et les caractéristiques de la crépine (diamètre des ouvertures de $7 \mathrm{~mm}$ et vitesse d'aspiration au niveau des ouvertures de $0,07 \mathrm{~m} \mathrm{~s}^{-1}$ ), on estime que le diamètre maximal des sédiments pouvant être aspirés par le bas de la crépine est de $0,28 \mathrm{~mm}$, alors que le diamètre maximal d'une particule aspirée par le haut n'est limité que par la dimension des ouvertures, i.e. $7 \mathrm{~mm}$. Ainsi, le système d'aspiration pourrait échantillonner entre $92 \%$ $(0,28 \mathrm{~mm})$ et $100 \%(7 \mathrm{~mm})$ des particules du sol de surface. Toutefois, comme la granulométrie des sédiments transportés par le ruissellement est plus fine que celle du sol de surface, l'échantillonneur est vraisemblablement en mesure de prélever toutes les particules en suspension dans le contenant de rétention.

Bien que la trappe à sédiments ait été conçue pour évaluer les pertes de contaminants par la fraction grossière des sédiments, le fait que l'ensemble des sédiments soit aspiré par l'échantillonneur rend superflue l'incorporation de cette donnée dans l'évaluation de la perte totale. La figure 4 montre que les sédiments déposés dans la trappe sont plus grossiers que ceux prélevés par l'échantillonneur car les particules plus fines restées en suspension ont été évacuées par le drain d'évacuation.

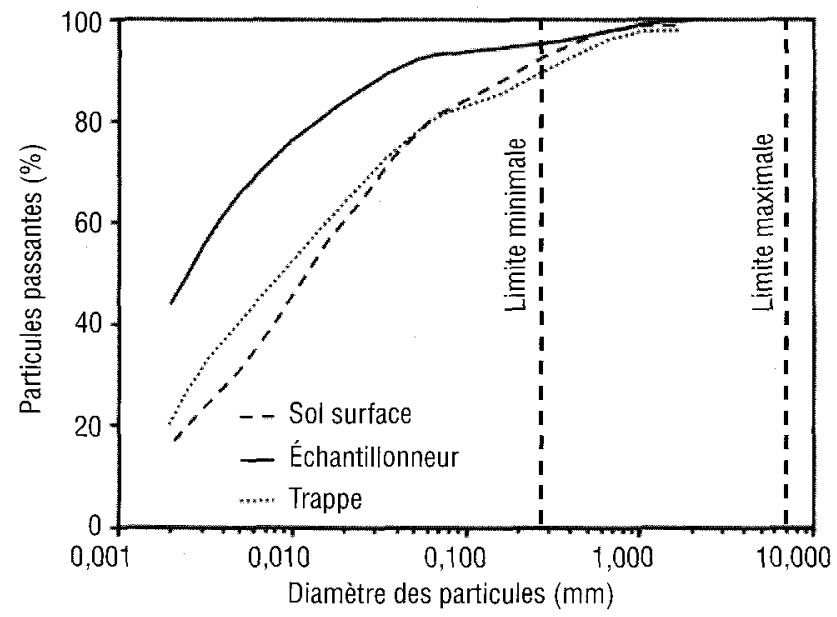

Figure 4 Courbes granulométriques du sol de surface et des sédiments dans le champ 58 (septembre 2001).

Particle size curves of topsoil and sediments in field plot no. 58 (September 2001). 
Une mauvaise représentativité de l'échantillon composé pourrait provoquer une imprécision sur la masse totale de sédiments exportés du champ. En effet, il est difficile de remettre les sédiments en suspension lors du prélèvement de l'eau dans le contenant de l'échantillonneur. Malgré une agitation préalable, une partie des sédiments grossiers peut se déposer dans le contenant avant le prélèvement de l'échantillon. Par exemple, en considérant une hauteur d'eau de $12 \mathrm{~cm}$ (contenant à moitié plein), les particules de $0,28 \mathrm{~mm}$ prennent 2,6 s à sédimenter, et celles de $0,1 \mathrm{~mm}$ prennent $21 \mathrm{~s}$. Comme la procédure requiert environ $10 \mathrm{~s}$ entre l'agitation et le prélèvement, une fraction des sédiments grossiers peut se redéposer et causer une sous-estimation de la masse totale de sédiments érodés du champ. Cette difficulté de remise en suspension des sédiments avant la prise des échantillons a été mentionnée par CHOW et al. (2000).

\section{4 - CONCLUSIONS}

Suite à deux années d'opération des systèmes automatiques de mesure de ruissellement et de drainage sur neuf parcelles expérimentales, les observations suivantes peuvent être faites. Le système automatique de mesure de ruissellement a permis la détermination des masses de contaminants agricoles quittant les parcelles par ruissellement du début de la fonte nivale jusqu'à tard l'automne. L'opération de ces systèmes a requis une présence fréquente sur le site, particulièrement durant la fonte nivale où il y a formation de glace. Ce système a permis un échantillonnage des formes solubles et particulaires de $P$ parvenant à l'exutoire de la parcelle. Des installations futures devraient prévoir des trappes à sédiments en fibre de verre, plastique ou acier de façon à éviter les fuites. La présence de courant électrique sur le site permettrait l'utilisation d'appareils fonctionnant sur le réseau et la réduction du temps requis pour l'opération des systèmes et aussi d'installer du chauffage pour prévenir la formation de glace au niveau du déversoir. Pour les situations où le débit de ruissellement excéderait la capacité des déversoirs de $10^{\circ}$ durant une partie de l'événement, il n'y aurait perte d'information de volume et de débit que pour cette période de débordement.

Parce que les débits de drainage sont plus réguliers et qu'il n'y a pas de sédiments ni de gel au niveau des drains, le système de mesure de drainage souterrain est plus simple et son opération a présenté moins de difficultés. Le plus grand obstacle à la mesure du drainage souterrain a été l'inondation printanière perturbant les systèmes de mesure. Le courant électrique permettrait l'installation de pompe dans le puits de captage afin d'éviter ce problème.

La répartition des pertes de $P$ entre le ruissellement et le drainage illustre l'importance de quantifier les pertes de contaminants agricoles en provenance de ces deux composantes de l'écoulement hydrique. La grande fraction du ruissellement durant la fonte nivale (89\%) a démontré la nécessité de mesures de contaminants agricoles durant cette période en plus de celles des périodes estivales et automnales. 


\section{REMERCIEMENTS}

Les auteurs remercient le Fonds de Recherche sur la Nature et les Technologies du Gouvernement du Québec (FQRNT-IRDA Actions concertées) pour le soutien financier, les étudiants et étudiantes qui ont participés au projet, ainsi que les employés de l'Institut de Recherche et de Développement en Agroenvironnement pour leur aide précieuse lors de l'installation et pour l'entretien des dispositifs expérimentaux.

\section{RÉFÉRENCES BIBLIOGRAPHIQUES}

ALLEN R.G., 2000. REF-ET Reference evapotranspiration calculator, Version 2.01.17. University of Idaho. www.kimberly.uidaho.edu/ref-et/

APHA, 1985a. Persulfate digestion method. In "Standard methods for the examination of waste and waster water", $16^{\mathrm{e}}$ édition. Greenberg et al. (Éds), American Public Health Association p. 444.

APHA, 1985b. Total suspended solids dried at $103-105^{\circ} \mathrm{C}$. In "Standard methods for the examination of waste and waster water", $16^{\mathrm{e}}$ édition. Greenberg et al. (Éds), American Public Health Association, pp. 93-94.

CHOW T. L., H. W. REES et J. MONTEITH, 2000. Seasonal distribution of runoff and soil loss under four tillage treatments in the upper St. John River valley New Brunswick. Canada. Can. J. Soil Sci. 80: 649-660.

ENVIRONNEMENT CANADA, 2004. Normales climatiques pour le Canada 19712000: Station de Scott, P.Q. Site web: Www.msc-smc.ec.gc.ca/climate.

FASSNACHT S.R., E.D. SOULIS et N. KOUWEN, 1999. Shape characteristics of freshly fallen snowflakes and their short-term changes. Interactions Between the Cryosphere, Climate and Greenhouse Gases 256: 111-112.

GANGBAZO G., A. R. PESANT, D. CÔTÉ, G. M. BARNETT et D. CLUIS, 1997. Spring runoff and drainage $N$ and $P$ losses from hog manured corn. Journal of American Water Resources Association 33(2): 405411.
GIROUX M. et T.S. TRAN, 1996. Critères agronomiques et environnementaux liés à la disponibilité, la solubilité et la saturation en phosphore des sols agricoles du Québec. Agrosol 9 (2): 51-57.

GUPTA R.S., 1997. Environmental engineering and science, An Introduction. Governement Institutes, Rockville, MD.

HANSEN N.C., S.C. GUPTA et J.F. MONCLIEF, 2000. Snowmelt runoff, sediment, and phosphorus losses under three different tillage systems. Soil Tillage Rechearch 57: 93-100.

KUYKENDALL H. A., M. L. CABRERA, C. S. HOVELAND, M. A. MCCANN et L. T. WEST, 1999. Stocking method effects on nutrient runoff from pasture fertilized with broiler litter. J. Environ. Qual. 28: 1886-1890.

JAMIESON A., C.A. MADRAMOOTOO and P. ENRIGHT, 2003. Phosphorus losses in surface and subsurface runoff from a snowmelt event on an agricultural field in Quebec. Canadian Biosystems Engineering 45: 1.1-1.7 version en ligne www.engr.usask.ca/societies/csae/journalcoverforweb2003.pdf

LAPP P., C. A. MADRAMOOTOO, P. ENRIGHT, F. PAPINEAU et J. PERRONE, 1998. Water quality of an intensive agricultural watershed in Quebec. Journal of the American Water Resources Association 34(2): 427-437.

MABIT L., M. R. LAVERDIÈRE et C. BERNARD, 2002. L'érosion hydrique: méthodes et études de cas dans le nord de la France. Cahiers Agriculture 11: 195-206. 
MONFET J., 1979. Évaluation du coefficient de ruissellement à l'aide de la méthode SCS modifiée., Gouvernement du Québec, Ministère des richesses naturelles, Service de l'hydrologie, Québec, Canada. Publication HP-53: 35.

PLAMONDON A.P., M. PRÉVOST, R.C. NAUD, 1984. Accumulation et fonte de la neige en milieux boisé et déboisé. Géogr. phys. et Quaternaire 38: 27-35.

ROMPRÉ M., 1985. Cartographie des sols, Station de recherché de St-Lambert de Lévis. Ministère de l'Agriculture, des Pêcheries et de l'Alimentation du Québec. Québec. Canada.

SHELDRICK B. H. et C. WANG, 1993. Particle-size distribution. In "Soll sampling and methods of analysis", M.R. Carter.
Boca Raton, FL. Lewis Publishers: pp. 499-511.

STONE K. C., P. G. HUNT, J. M. NOVAK, M. H. JOHNSON et D. W. WATTS, 2000. Flow-proportional, time-composite, and grab sample estimation of nitrogen export from an eastern coastal plain watershed. Transactions of ASAE 43: 281-290.

TECHNICON, 1973. Total inorganic phosphate in water and sea water. Industrial method 93-70W, Technicon Industrial systems, Tarrytown, NY.

WILLIAMS G.P., 1959. Evaporation from snow covers in eastern Ontario. National Research Council, Ottawa, ON. Research paper, Division of Building Research, 0077-5525, № 73. 